\title{
Factores pronósticos en la diverticulitis aguda
}

\section{Predictors of acute diverticulitis}

\author{
Guillermo R. Guevara-Morales ${ }^{1 *}$ y Juan C. Castellanos-Juárez ${ }^{2}$
}

${ }^{1}$ Servicio de Cirugía General; ${ }^{2}$ Servicio de Coloproctología. Hospital Regional de Veracruz, Instituto de Seguridad y Servicios Sociales de los Trabajadores del Estado, Veracruz, Ver. México

A los editores

Leímos con interés el trabajo presentado por el Dr. García-Gómez, et al. ${ }^{1}$ en el que se analiza el valor pronóstico de la presencia de burbujas de aire libre pericólico detectadas por tomografía computada en la diverticulitis aguda (DA). Sabemos que el manejo de la DA es controvertido, y aunque sus resultados son sumamente interesantes, creemos pertinente realizar los siguientes comentarios:

- No se especifican el tiempo de seguimiento de los pacientes, la tasa de reintervención quirúrgica ni la descripción detallada de las complicaciones, lo cual consideramos es importante cuando se investiga el pronóstico de un factor en la DA.

- En la discusión, los autores comentan como un dato relevante los valores de leucocitos y la relación con el éxito del manejo conservador; no obstante, su función como factor predictivo no es del todo clara. Por el contrario, existen puntajes con significado pronóstico para la DA con peritonitis, como APACHE II, el índice de Mannheim, PSS (Left CoIonic Peritonitis Severity Score) y DDPS (Cleveland Clinic Diverticular Disease Propensity Score), e incluso algunos marcadores como la proteína $C$ reactiva, que son más específicos que el recuento de leucocitos como predictor en la $\mathrm{DA}^{2-4}$.

Los autores concluyen que la presencia de burbujas de aire libre pericólico en pacientes con DA puede ser considerada de valor pronóstico, si bien cumple con el objetivo de su estudio. Se han descrito múltiples factores que guardan estrecha relación con la morbimortalidad en pacientes con DA, tales como el riesgo quirúrgico ASA (clasificación del estado físico de la american society of anesthesiology), el tiempo de evolución, el inmunocompromiso, el uso de antiinflamatorios no esteroideos, la indicación y el tipo de cirugía, y el grado de contaminación del procedimiento, entre otros, que permiten identificar el riesgo de la población estudiada y permitirían conocer la influencia sobre la duración de la estancia intrahospitalaria, la tolerancia a la vía oral y otras variables estudiadas ${ }^{5}$.

No sin antes felicitarlos por la autenticidad y los resultados de su estudio, esperamos que los comentarios realizados sean de utilidad en un futuro a fin de validar la utilidad de este hallazgo tomográfico en los pacientes con DA de colon.

\section{Conflicto de intereses}

Los autores declaran no tener conflicto de intereses.

\section{Financiamiento}

El financiamiento fue realizado por los autores.

\section{Bibliografía}

1. García-Gómez MA, Belmonte-Montes C, Cosme-Reyes C, Preben Aguirre-García M. Valor pronóstico de la presencia de burbujas de aire libre pericólico detectadas por tomografía computada en diverticulitis aguda. Cir Cir. 2017;85:471-7.

2. Biondo S, Ramos E, Fraccalvieri D, Kreisler E, Rague JM, Jaurrieta $E$. Comparative study of left colonic Peritonitis Severity Score and Mannheim Peritonitis Index. Br J Surg. 2006;93:616-22.

3. Aydin HN, Tekkis PP, Remzi FH, Constantinides V, Fazio VW. Evaluation of the risk of a nonrestorative resection for the treatment of diverticular disease: The Cleveland Clinic diverticular disease propensity score. Dis Colon Rectum. 2006;49:629-39.

4. Tan JPL, Barazanchi AWH, Singh PP, Hill AG, Maccormick AD. Predictors of acute diverticulitis severity: a systematic review. Int J Surg. 2016; 26:43-52.

5. Roig JV, Salvador A, Frasson M, Cantos M, Villodre C, Balciscueta Z, et al. Tratamiento quirúrgico de la diverticulitis aguda. Estudio retrospectivo multicéntrico. Cir Esp. 2016:94:569-77.

\section{Correspondencia:}

*Guillermo R. Guevara-Morales

Av. Díaz Mirón esquina Sayula, s/n 